\title{
BMJ Open Association between relocation and changes in cardiometabolic risk factors: a longitudinal study in tsunami survivors of the 2011 Great East Japan Earthquake
}

\author{
Shuko Takahashi, ${ }^{1,2}$ Motoyuki Nakamura, ${ }^{3}$ Yuki Yonekura, ${ }^{2}$ Kozo Tanno, ${ }^{2}$ \\ Kiyomi Sakata, ${ }^{2}$ Akira Ogawa, ${ }^{4}$ Seiichiro Kobayashi ${ }^{5}$
}

To cite: Takahashi S, Nakamura M, Yonekura Y, et al. Association between relocation and changes in cardiometabolic risk factors: a longitudinal study in tsunami survivors of the 2011 Great East Japan Earthquake. BMJ Open 2016;6:e011291.

doi:10.1136/bmjopen-2016011291

- Prepublication history and additional material is available. To view please visit the journal (http://dx.doi.org/ 10.1136/bmjopen-2016011291).

Received 27 January 2016 Revised 19 April 2016 Accepted 26 April 2016

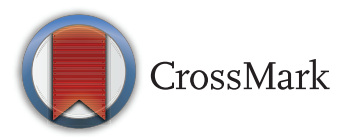

For numbered affiliations see end of article.

Correspondence to Dr Shuko Takahashi; shutakahashi-iwt@umin.ac.jp

\section{ABSTRACT}

Objectives: The aim of this study is to determine changes in atherosclerotic cardiovascular risk factors with and without serious disaster-related mental and socioeconomic problems represented by relocation (REL).

Design: A longitudinal survey.

Setting: Multiphasic health check-ups for the general population affected by the 2011 Great East Japan Earthquake and Tsunami.

Participants: A total 6528 disaster survivors in heavily tsunami-damaged municipalities were recruited. Two sequential surveys were conducted and the data were analysed.

Main outcome measures: Multiphasic health check-ups including investigation of lifestyle and psychological and socioeconomic measures were performed in two sequential phases (8 and 18 months) after the disaster for tsunami survivors with REL $(n=3160)$ and without REL ( $n=3368)$. Longitudinal changes in cardiometabolic risk factors between the two phases were compared in the REL and non-REL groups.

Results: In sex/age-adjusted analysis, we found increases in body weight and waist circumference between the two phases that were significantly greater in the REL group than in the non-REL group (body weight: $+0.31(0.23 \sim 0.39)$ versus -0.24 $(-0.32 \sim 0.16) \mathrm{kg}, \mathrm{p}<0.001$; waist circumference: +0.58 (0.48 0.68) versus $+0.05(-0.05 \sim 0.15) \mathrm{cm}, \mathrm{p}<0.001))$. $A$ decrease in serum HDLC levels was found and again was significantly greater in the REL group than in the non-REL group $(-0.65(-0.96 \sim-0.34)$ versus -0.09 $(-0.39 \sim 0.21) \mathrm{mg} / \mathrm{dL}, \mathrm{p}=0.009)$. In addition, deterioration in physical activity, mental health and socioeconomic status was more prevalent in the REL group than in the non-REL group (all $p<0.001$ ).

Conclusions: This study suggests that relocation after the devastating tsunami was related to weight gain and decreasing HDLC among survivors, and this change was associated with prolonged psychological distress and socioeconomic problems after the disaster.

\section{Strengths and limitations of this study}

- In this longitudinal survey, changes in cardiometabolic risk factors were determined in the general population affected by the 2011 Japan disaster.

- Several types of self-reported questionnaire concerning lifestyle and psychological and socioeconomic parameters were used.

- The survey was performed in two sequential phases (8 and 18 months) after the disaster.

- Sex-adjusted and age-adjusted changes between the two phases were compared in participants with relocation $(n=3160)$ and those without relocation $(\mathrm{n}=3368)$.

- It was not determined in this study whether there was any overall increase in the incidence of atherosclerotic cardiovascular incidents during the study period.

\section{INTRODUCTION}

A 9.0 magnitude earthquake, named the 2011 Great East Japan Earthquake, and a subsequent catastrophic tsunami struck the northeast region of Honshu, Japan on 11 March 2011. The devastating tsunami struck coastal towns and cities and destroyed a large number of local communities (see online supplementary figure S1). In Iwate prefecture, one of the most heavily damaged areas, about 5800 residents drowned or went missing on the day the tsunami struck. ${ }^{1}$ Tsunami survivors whose homes were severely damaged were forced to evacuate to community centres or crowded school gymnasiums soon after the disaster. Even several years after the disaster, the local government has placed restrictions on where survivors can rebuild houses in order to protect against future tsunami events. Some survivors could 
not afford to build new houses, so they were forced to move into small temporary housing (see online supplementary figure S2-3) and suffered psychological and socioeconomic problems. ${ }^{2}$ Those survivors had to move to different residences several times in the aftermath period. On the other hand, residents whose homes were not destroyed by the tsunami could continue with their usual lifestyle after the disaster. These survivors did not have to relocate during the postdisaster period.

Previous studies have shown an increase in the incidence of several types of cardiovascular diseases after natural disasters, ${ }^{3-5}$ and several studies have shown increased numbers of patients with acute myocardial infarction, sudden cardiac death, heart failure and stroke in the present disaster area. ${ }^{6-9}$ However, the detailed mechanisms underlying the increased posttsunami incidence of these atherosclerotic cardiovascular events have not yet been fully determined. Several reports have suggested that one of the reasons for this increased incidence in disaster areas may be worsening atherosclerotic cardiovascular risk profiles combined with postdisaster psychological distress. ${ }^{10-12}$

Therefore, the purpose of this study was to determine the longitudinal changes in several atherosclerotic cardiovascular risk factors after the disaster among community-dwelling adults with and without serious disaster-related mental and socioeconomic problems represented by relocation (REL).

\section{MATERIALS AND METHODS \\ Study participants}

Adult participants were recruited from the general population in the heavily tsunami-damaged cities of Yamada, Otsuchi and Rikuzentakata near the seacoast of the southern part of Iwate Prefecture (figure 1). ${ }^{13}$ This cohort study project was named RIAS (Research project for prospective Investigation of health problems Among Survivors of the 2011 Great East Japan Earthquake and Tsunami), and the purpose of this study and the

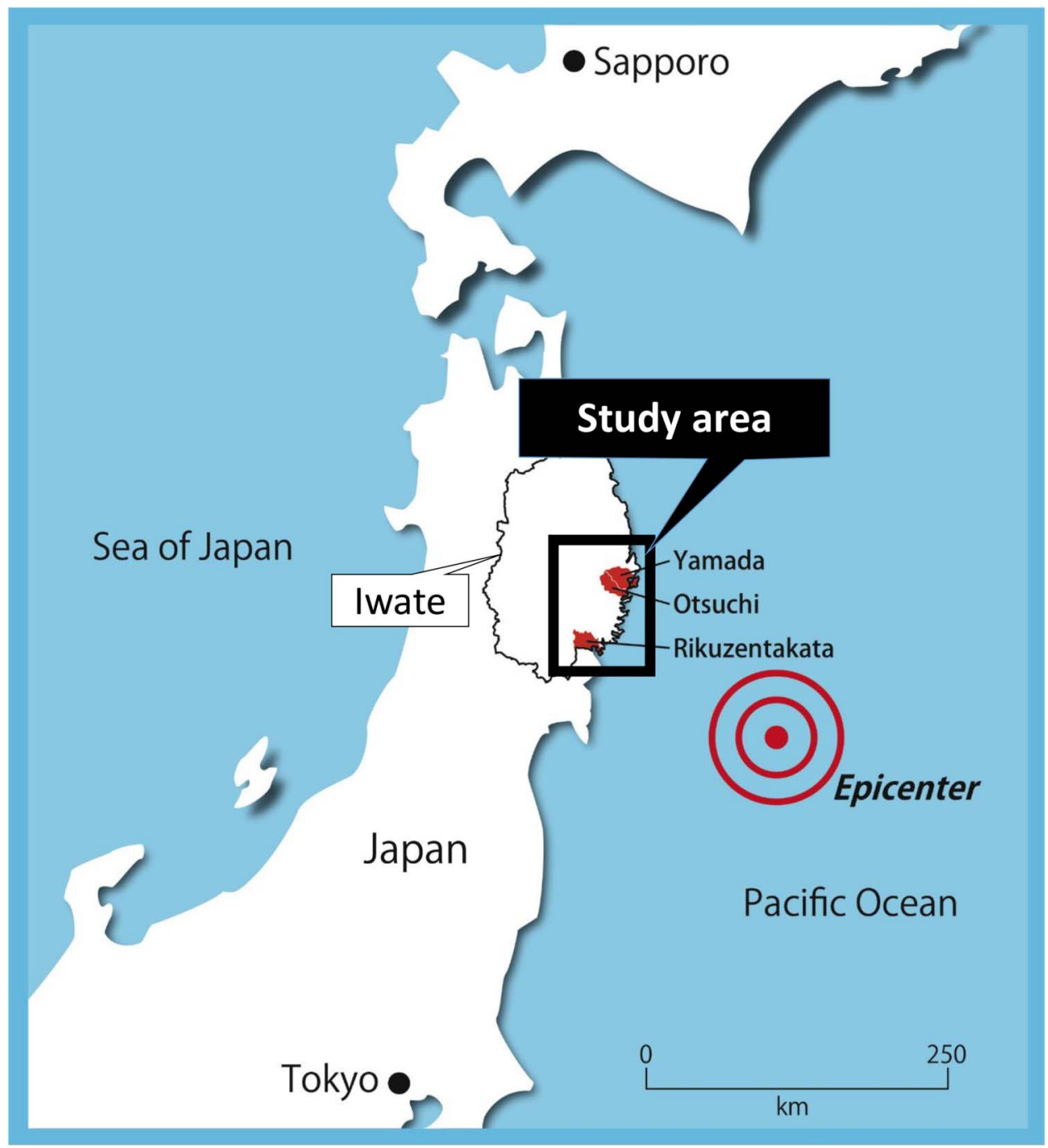

Figure 1 Map of the study area. The black square shows the study area along the Pacific Ocean coast that was affected by the tsunami. The municipalities included in our study were Yamada, Otsuchi and Rikuzentakata. The epicentre of the earthquake is marked as a dot. 
detailed methods of subject recruitment were reported in our previous paper. ${ }^{14}$

In brief, after a public announcement, we sent out notifications of the health survey and several types of structural questionnaire (lifestyle, mental health and socioeconomic status) to all residents aged 18 years or older (12 772 people in Yamada, 11411 in Otsuchi and 18648 in Rikuzentakata: total 42 831). After this, a total of 10558 residents attended the study site, and 10196 participants (participation rate of about 24\%) finally agreed to participate after giving written informed consent (figure 2). The initial survey consisted of anthropometric, clinical and physiological measures, and self-reported questionnaires were given during the postdisaster phase (mean period of 8 months after the disaster=phase 1) in the three municipalities.

The second postdisaster survey employed similar study items (anthropometric, clinical and physiological measures, and self-reported questionnaires) and was conducted in the same districts in 2012 (mean period of 18 months after the disaster=phase 2). At the phase 2 examination, 2689 participants who participated in the phase 1 survey did not attend. After excluding persons who did not completely respond to the follow-up questionnaires $(\mathrm{n}=979)$, we finally analysed data from 6528 participants (2499 males and 4029 females). The rights and welfare of participants in this study were protected by the ethical guidelines outlined in the Declaration of Helsinki. The research plan was deliberated and approved by the Ethics Committee of Iwate Medical University (approval no. H23-69).

\section{Anthropometric data and blood pressure}

Body weight $(\mathrm{kg})$ was measured using digital scales (AD-6400, A\&D Co., Tokyo, Japan; BWB-200, Tanita Co.,
Tokyo, Japan) with an accuracy of $\pm 0.1 \mathrm{~kg}$ and with participants wearing light clothing and no shoes. Height was measured using digital scales (AD-6121A, A\&D Co., Tokyo, Japan; YG200D, Yagami INC, Nagoya, Japan). Body mass index (BMI; $\mathrm{kg} / \mathrm{m}^{2}$ ) was calculated by dividing body weight $(\mathrm{kg})$ by height $(\mathrm{m})^{2}$. Waist circumference was measured standing up using tape measure lines.

Experienced research staff measured systemic blood pressure using an automatic digital device (BP-103i II, Omron Colin Co, Tokyo, Japan) with the subject seated after at least 5 min of rest. Each measurement was performed twice, and mean values for systolic blood pressure (SBP; $\mathrm{mm} \mathrm{Hg}$ ) and diastolic BP (DBP; $\mathrm{mm} \mathrm{Hg}$ ) were calculated. Mean values were used for statistical analysis.

\section{Biochemical analyses}

Blood samples were drawn from the antecubital vein while participants were seated. The samples were transported to a laboratory (Iwate Health Service Association) and were analysed on the same day. We determined serum levels of total cholesterol $(\mathrm{TC} ; \mathrm{mg} / \mathrm{dL})$, high-density lipoprotein cholesterol (HDLC; $\mathrm{mg} / \mathrm{dL}$ ), low-density lipoprotein cholesterol (LDLC; $\mathrm{mg} / \mathrm{dL}$ ) and triglyceride (TG; $\mathrm{mg} / \mathrm{dL}$ ). Serum TC levels were determined by an enzymatic assay, and serum concentrations of HDLC and LDLC were determined by a direct quantitative assay. The quality of TC and HDLC measurements was controlled by the programme of the Centers for Disease Control in the USA. Serum TG concentration was determined by an enzyme colorimetric assay. We examined serum creatinine levels using an enzymatic assay. All of the above biochemical data were determined by using an automated analyser (Hitachi 7700, Tokyo, Japan). Non-high-density lipoprotein cholesterol (non-HDLC; mg/dL) was calculated by

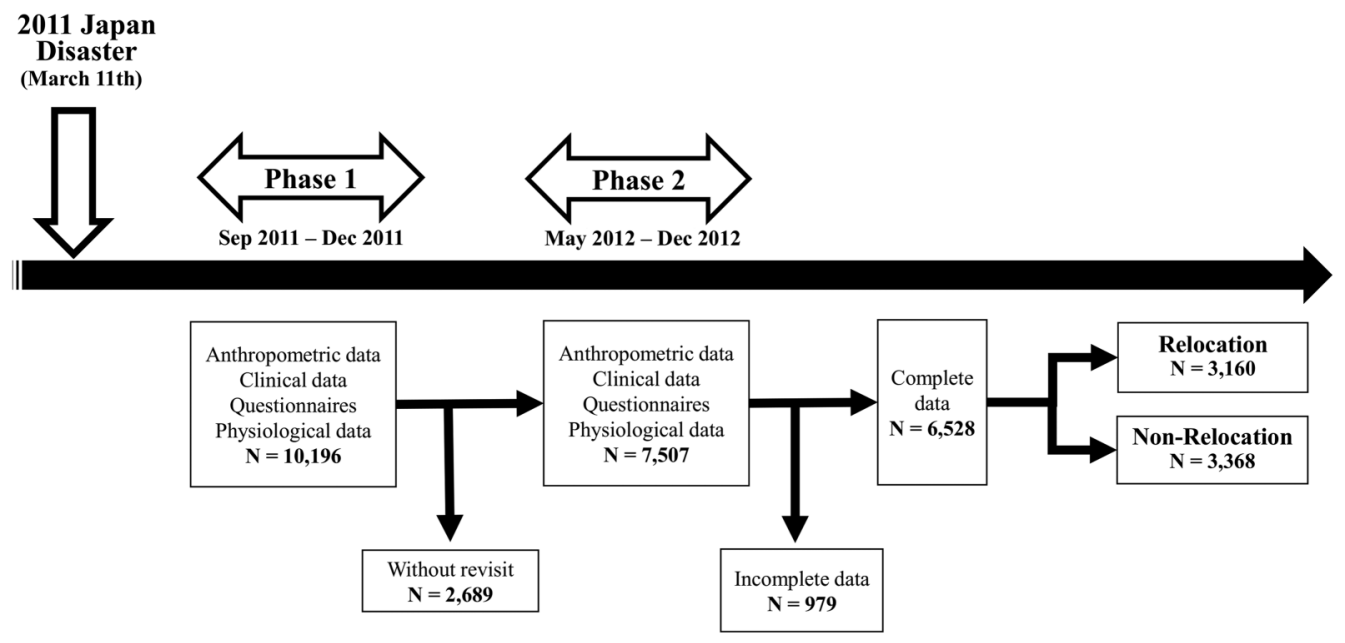

Figure 2 Flow chart of the study phase and the procedure used to select participants for the study. The survey in 2011 (phase 1) was conducted 6 months after the disaster (from September to December 2011). The original cohort consisted of 10196 participants in phase 1. The next survey (phase 2) was carried out 18 months after the disaster (from September to December 2012). The average period between phase 1 and phase 2 was 11 months. We excluded a total of 2689 persons who did not participate in the phase 2 survey and 979 persons who lacked at least one variable used for analysis. We finally used data from 6528 participants for analysis. 
subtracting HDLG from TC. LDL/HDL cholesterol ratio (LH ratio) was calculated by dividing LDLC $(\mathrm{mg} / \mathrm{dL})$ by HDLC ( $\mathrm{mg} / \mathrm{dL})$. We examined glycosylated haemoglobin (HbAlc, \%) levels by high-performance liquid chromatography using an automated analyzer (Tosoh HLC-723G7, Japan). The value for HbA1c (Japan Diabetes Society; JDS) was estimated as an equivalent value of the National Glycohemoglobin Standardization Program (NGSP) calculated by the formula HbAlc (NGSP) $=\mathrm{HbAlc}($ JDS $)+0.4 .^{15}$

\section{Self-report questionnaire}

In the phase 1 survey, several types of self-report questionnaires were administered to assess lifestyle, mental health and socioeconomic status of participants after the disaster. Relocation (REL) status was assessed by the question "How many times did you change your house (including a shelter evacuation) after the disaster?" According to the answers, we classified REL status into two categories: REL group ( $\geq$ once) and non-REL group. The REL group would be survivors who lost their homes due to the tsunami moved to evacuation centres or to family and friend's houses after the disaster. Later, most of them moved to temporary housing. Smoking status (current, past smoker or non-smoker) and alcohol drinking status (no drinker, occasional drinker or daily drinker) were also ascertained by selfreported questionnaires. Smoking status was classified into current smokers or non-smokers. For alcohol drinking status, we classified participants who marked occasional or daily as drinkers and participants who marked not alcohol drinker as non-drinkers. In order to assess daily physical activity after the disaster, we asked three questions regarding the quality, frequency and duration of daily physical activity, with participants divided into low or normal levels as per previous reports. ${ }^{16} 17$

Levels of psychological distress after the disaster were assessed by the Kessler Psychological Distress Scale (K6) questionnaires. ${ }^{18}$ On the basis of a previous study using the $\mathrm{K} 6$ test among Japanese participants that consisted of six self-reported items, participants were classified into those with psychological distress (scores of 5-24) and those with no psychological distress (scores of $0-4) .{ }^{19}$ Sleep difficulty was assessed using the Athens Insomnia Scale. ${ }^{20}$ On the basis of a previous study using the Athens Insomnia Scale, participants were classified as those with insomnia (scores of 6-24) and those with no insomnia (scores of $0-5){ }^{21}$

Employment status after the disaster was assessed by a self-reported questionnaire. On the basis of their answers, participants were categorised into two groups: unemployment or not. Economic status was also assessed by asking the question "How do you feel about your current economic situation?" Participants were asked to choose one of the four answers (very serious, serious, slightly serious or usual). On the basis of their answers, participants were categorised into two groups: economic deprivation (very serious, serious and slightly serious) or usual.
We asked about medical history of diabetes mellitus, dyslipidaemia and hypertension in the questionnaires, and the participants were categorised into two groups (yes or no) for diabetes mellitus, dyslipidaemia and hypertension.

\section{Statistical analysis}

Several baseline characteristics and changes in cardiometabolic parameters between phase 1 and phase 2 were compared in the REL group and the non-REL group. In crude analysis, the differences were tested by a $\chi^{2}$ test for categorical variables and Student's $t$ test for continuous variables. Categorical variables were shown as the proportion (\%) and continuous variables were shown as the mean and 95\% CI. In sex-adjusted and age-adjusted analysis, the differences were tested by logistic regression analysis for categorical variables and by analysis of covariance for continuous variables. Categorical variables were shown as the proportion (\%) and continuous variables were shown as the mean and 95\% CI. The changes in anthropometric and clinical characteristics between the two phases were calculated by subtracting variables in phase 1 from variables in phase 2. In crude analysis, mean changes in several variables were compared between the REL group and the non-REL group by using Student's t test. In sex-adjusted and age-adjusted analysis, adjusted mean changes were compared between the REL group and the non-REL group by using analysis of covariance and were shown as the adjusted mean \pm SE. In addition, to determine whether REL was an independent variable to predict changes in body weight, stepwise multiple linear regression analysis was used to adjust for potential risk factors (sex, age, weight, SBP, smoking status, physical activity, psychological distress, unemployment and economic deprivation).

All $p$ values were based on two-sided tests, and $p$ values of less than 0.05 were considered statistically significant. The Statistical Package for Social Sciences (SPSS) V.19.0 (IBM) was used for all analyses.

\section{RESULTS}

Table 1 shows the clinical characteristics of participants in terms of demographic and anthropometric data, clinical characteristics, lifestyle, mental health and socioeconomic status for the REL and non-REL groups at the 2011 baseline survey (phase 1). The final numbers of participants were 3160 in the REL group and 3368 in the non-REL group. The percentage of men was not significantly different between the two groups $(38 \%$ for REL vs $38 \%$ for non-REL; $p=0.972$ ). In crude analysis, the average ages were 61 years for the REL group and 63 years for the non-REL group $(\mathrm{p}<0.001)$. Body weight in the REL group was significantly higher than that in the non-REL group ( 58.7 vs $57.8 \mathrm{~kg}$; $\mathrm{p}=0.001$ ). Waist circumference was not significantly different between the two groups $(\mathrm{p}=0.238)$. 
Table 1 Comparison of baseline characteristics of the 2011 baseline survey (phase 1) between the REL group and the non-REL group

\begin{tabular}{|c|c|c|c|c|c|c|}
\hline & \multicolumn{3}{|l|}{ Crude } & \multicolumn{3}{|l|}{ Adjusted } \\
\hline & REL (N=3160) & non-REL ( $\mathrm{N}=3368)$ & p Value & REL (N=3160) & non-REL ( $\mathrm{N}=3368)$ & p Value \\
\hline \multicolumn{7}{|l|}{ Demographic data } \\
\hline Sex (male) & $38 \%$ & $38 \%$ & 0.972 & & & \\
\hline Age (years) & $61.2(60.7-61.7)$ & $63.4(62.9-63.8)$ & $<0.001$ & & & \\
\hline \multicolumn{7}{|l|}{ Anthropometric data } \\
\hline Body weight $(\mathrm{kg})$ & $58.7(58.3-59.1)$ & $57.8(57.4-58.1)$ & 0.001 & $59.9(59.6-60.3)$ & $59.4(59.1-59.7)$ & 0.016 \\
\hline Waist circumference $(\mathrm{cm})$ & $83.1(82.8-83.5)$ & $82.9(82.6-83.2)$ & 0.238 & 83.6 (83.3-83.9) & $83.2(82.9-83.5)$ & 0.073 \\
\hline \multicolumn{7}{|l|}{ Clinical data } \\
\hline SBP $(\mathrm{mm} \mathrm{Hg})$ & 125.3 (124.7-125.9) & $127.4(126.8-128.1)$ & $<0.001$ & $126.1(125.5-126.7)$ & $127.4(126.8-128.0)$ & 0.003 \\
\hline $\mathrm{DBP}(\mathrm{mm} \mathrm{Hg})$ & $74.0(73.6-74.3)$ & $74.5(74.2-74.9)$ & 0.030 & $74.5(74.1-74.9)$ & $74.9(74.5-75.3)$ & 0.108 \\
\hline $\mathrm{TC}(\mathrm{mg} / \mathrm{dL})$ & 205.9 (204.7-207.1) & 204.4 (203.2-205.6) & 0.084 & 204.4 (203.1-205.6) & $202.9(201.8-204.1)$ & 0.099 \\
\hline HDLC (mg/dL) & $63.9(63.3-64.5)$ & $63.7(63.1-64.3)$ & 0.666 & $62.9(62.3-63.5)$ & $63.0(62.4-63.6)$ & 0.822 \\
\hline $\operatorname{LDLC}(\mathrm{mg} / \mathrm{dL})$ & $118.9(117.9-120.0)$ & $117.5(116.4-118.5)$ & 0.049 & $118.0(117.0-119.1)$ & $116.4(115.4-117.4)$ & 0.029 \\
\hline non-HDLC (mg/dL) & $142.0(140.7-143.2)$ & $140.7(139.5-141.9)$ & 0.136 & $141.5(140.2-142.7)$ & $140.0(138.7-141.2)$ & 0.089 \\
\hline LH ratio & $2.01(1.98-2.04)$ & $1.99(1.96-2.02)$ & 0.329 & $2.03(2.00-2.06)$ & $2.00(1.97-2.03)$ & 0.155 \\
\hline TG (mg/dL) & $140.0(136.9-143.0)$ & $137.2(134.3-140.0)$ & 0.188 & $142.9(139.9-145.9)$ & $140.3(137.3-143.2)$ & 0.215 \\
\hline $\mathrm{HbA} 1 \mathrm{c}(\%)$ & $5.67(5.65-5.69)$ & $5.68(5.66-5.70)$ & 0.528 & $5.69(5.67-5.71)$ & $5.68(5.66-5.70)$ & 0.483 \\
\hline Creatinine (mg/dL) & $0.70(0.70-0.71)$ & $0.71(0.70-0.72)$ & 0.330 & $0.71(0.70-0.72)$ & $0.73(0.73-0.74)$ & 0.798 \\
\hline \multicolumn{7}{|l|}{ Lifestyle } \\
\hline Current smokers & $16 \%$ & $12 \%$ & $<0.001$ & $12 \%$ & $10 \%$ & 0.051 \\
\hline Drinkers & $33 \%$ & $31 \%$ & 0.199 & $33 \%$ & $33 \%$ & 0.608 \\
\hline Low physical activity & $70 \%$ & $62 \%$ & $<0.001$ & $69 \%$ & $61 \%$ & $<0.001$ \\
\hline \multicolumn{7}{|l|}{ Mental health } \\
\hline Psychological distress & $47 \%$ & $37 \%$ & $<0.001$ & $46 \%$ & $36 \%$ & $<0.001$ \\
\hline Insomnia & $38 \%$ & $27 \%$ & $<0.001$ & $36 \%$ & $25 \%$ & $<0.001$ \\
\hline \multicolumn{7}{|l|}{ Socioeconomic status } \\
\hline Unemployment & $24 \%$ & $14 \%$ & $<0.001$ & $23 \%$ & $14 \%$ & $<0.001$ \\
\hline Economic deprivation & $59 \%$ & $43 \%$ & $<0.001$ & $59 \%$ & $44 \%$ & $<0.001$ \\
\hline \multicolumn{7}{|l|}{ Medical history } \\
\hline Diabetes mellitus & $8 \%$ & $6 \%$ & 0.061 & $7 \%$ & $6 \%$ & 0.008 \\
\hline Dyslipidaemia & $14 \%$ & $13 \%$ & 0.061 & $12 \%$ & $10 \%$ & 0.043 \\
\hline Hypertension & $34 \%$ & $34 \%$ & 0.219 & $32 \%$ & $29 \%$ & 0.017 \\
\hline
\end{tabular}

In the crude analysis, categorical variables are shown as proportion (\%) and continuous variables are shown as mean and $95 \% \mathrm{Cl}$.

In sex-adjusted and age-adjusted analysis, categorical variables are shown as adjusted proportion (\%) and continuous variables are shown as adjusted mean and $95 \% \mathrm{Cl}$.

DBP, diastolic blood pressure; HbA1c, glycosylated haemoglobin; HDLC, high-density lipoprotein cholesterol; LDLC, low-density lipoprotein cholesterol; LH ratio, LDL/HDL cholesterol ratio;

non-HDLC, non-high-density lipoprotein cholesterol; non-REL group, non-relocation group; REL group, relocation group; SBP, systolic blood pressure; TC, total cholesterol; TG, triglyceride. 
In sex/age-adjusted analysis, systemic blood pressure was significantly lower in the REL group than in the non-REL group (SBP, $\mathrm{p}=0.003$; DBP, $\mathrm{p}=0.108$ ). For the lipid panel, although there were no significant differences in baseline levels of TC, HDLG, non-HDLC, LH ratio and TG between the two groups, serum LDLC in the REL group was significantly higher than that in the non-REL group $(\mathrm{p}=0.029)$. Serum levels of HbA1c and creatinine were comparable between the two groups.

Although the adjusted proportion of current smokers in the REL group was higher than that in the non-REL group ( $12 \%$ vs $10 \%$; $\mathrm{p}=0.051$ ), there was no significant difference in the proportion of drinkers between the two groups (table 1). Daily physical activity assessed by the self-reported questionnaires was predominantly lower in the REL group ( $69 \%$ vs $61 \%$; $<<0.001)$. The percentage of participants with psychological distress was significantly higher in the REL group than in the non-REL group (K6 score higher than 5 points: $46 \%$ vs $36 \%: \mathrm{p}<0.001)$. Similarly, the frequency of insomnia (Athens Insomnia Scale $\geq 6$ points) was significantly higher in the REL group (36\% vs $25 \%$; $\mathrm{p}<0.001)$. The prevalence of unemployment or economic deprivation was significantly higher in the REL group (unemployment: $23 \%$ vs $14 \%, \mathrm{p}<0.001$; deprivation: $59 \%$ vs $44 \%$, $\mathrm{p}<0.001)$.

Although proportions of participants with a medical history of diabetes mellitus, dyslipidaemia and hypertension were not significantly different between the REL group and the non-REL group in crude analysis, adjusted proportions were higher in the REL group than in the non-REL group (diabetes mellitus, $\mathrm{p}=0.008$; dyslipidaemia, $\mathrm{p}=0.043$; hypertension, $\mathrm{p}=0.017$ ) .

Table 2 shows a comparison of crude and sex/ age-adjusted mean changes in anthropometric measurements and clinical characteristics between phase 1 and phase 2 for the REL and non-REL groups. The differences in crude changes in body weight $(+0.35 \mathrm{vs}-0.21 \mathrm{~kg}$; $\mathrm{p}<0.001)$ and waist circumference $(+0.57$ vs $+0.04 \mathrm{~cm}$; $\mathrm{p}<0.001)$ were significantly greater in the REL group than in the non-REL group. After adjustment for sex and age, these differences remained robust (body weight: $+0.31 \mathrm{~kg}$ in REL vs $-0.24 \mathrm{~kg}$ in non-REL, $\mathrm{p}<0.001$; waist circumference $: 0.58 \mathrm{~cm}$ in REL versus $+0.05 \mathrm{~cm}$ in non-REL, $\mathrm{p}<0.001$; table 2, figure $3 \mathrm{~A}, \mathrm{~B})$.

When multivariate regression analysis was performed to determine whether REL was an independent variable to predict changes in body weight between phases 1 and 2, this factor was the most powerful predictor for changes in body weight even after controlling for several confounding factors (table 3 ).

For other changes in the metabolic risk profile, the only significant difference between the two groups was in crude HDLG levels $(-0.75$ in REL vs $-0.17 \mathrm{mg} / \mathrm{dL}$ in non-REL, $\mathrm{p}=0.007$; table 2 ). The significance was apparent after sex/age-adjusted analysis $(-0.65 \mathrm{mg} / \mathrm{dL}$ in REL vs $-0.09 \mathrm{mg} / \mathrm{dL}$ in non-REL, $\mathrm{p}=0.009$; figure 3C). However, other clinical parameters such as blood

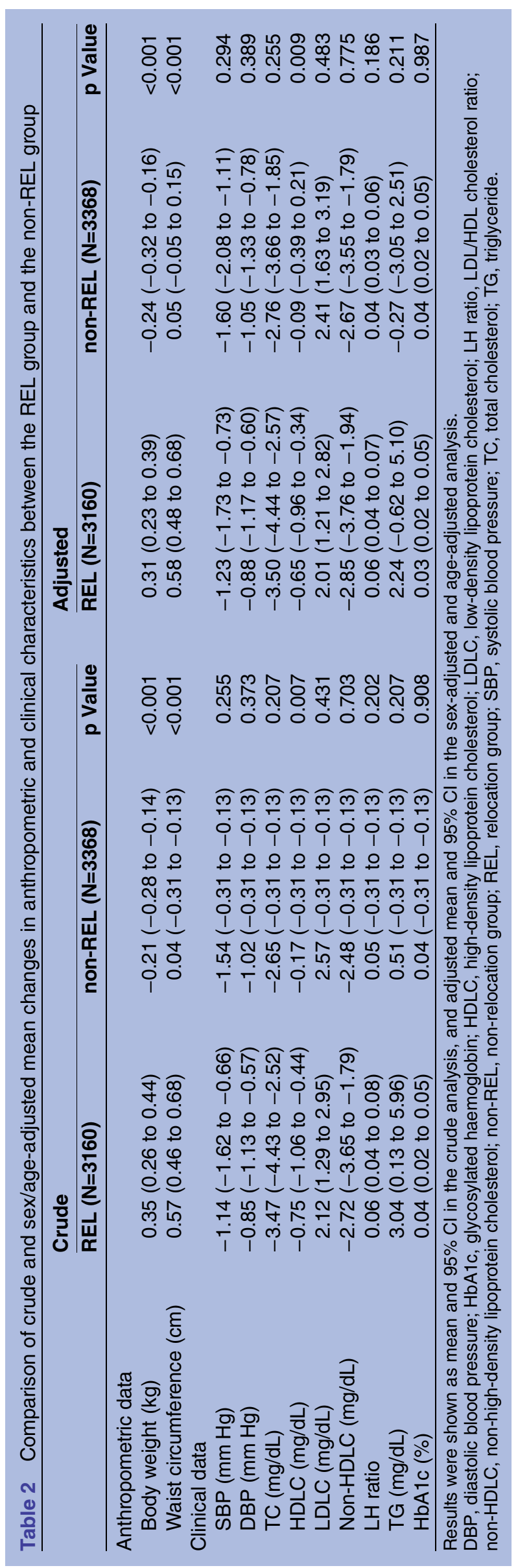



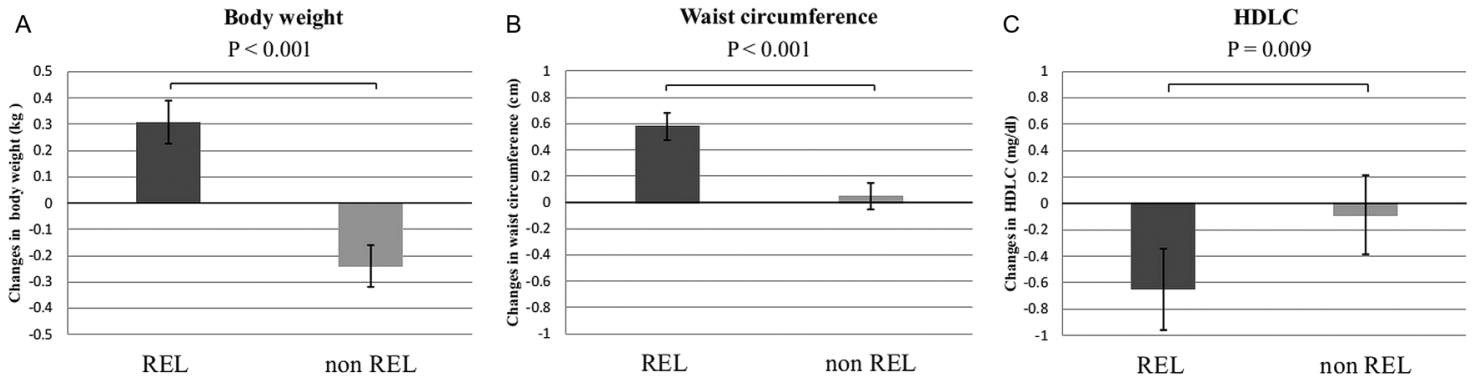

Figure 3 Comparison of sex-adjusted and age-adjusted mean changes in body weight (3A), waist circumference (3B) and serum HDLC level (3C) between phase 1 and phase 2 surveys for the REL and non-REL groups. Adjusted mean and 95\% Cl. HDLC, high-density lipoprotein cholesterol; REL, relocation group; non-REL, non-relocation group.

pressure and serum HbAlc did not differ significantly between the two phases (table 2).

\section{DISCUSSION}

The present study demonstrated that even more than 1 year after the devastating natural disaster, cardiometabolic risk factors such as body weight/waist circumference were increasing with deteriorating serum lipid profiles, especially HDLC, among survivors who had experienced serious disaster-related property damage as referenced by REL. These survivors had lost their homes because of the tsunami and had been forced to live in temporary housing (see online supplementary figure S2-3). In such cases, it is possible that those people had also lost family members, relatives and/or friends, property and jobs, and were more likely to be experiencing long-term psychological distress. To the best of our knowledge, this study is the first to determine longitudinal changes in several ACV risk factors combined with lifestyle and psychological and socioeconomic parameters in survivors of the devastating tsunami.

\section{Previous studies}

Several previous studies have shown short-term changes in anthropometric parameters, blood pressure, heart rate, lipids and glycaemic index after natural disasters in relatively small numbers of people in specific populations such as patients with hypertension, ${ }^{22-24}$ those with diabetes, $^{25}$ factory workers ${ }^{26}$ and disaster evacuees. ${ }^{27}$ However, there have been few studies in which the longterm impacts of a devastating tsunami on these parameters were examined in a large number of participants selected from the general population. Moreover, in previous studies, the impact of psychological distress and socioeconomic problems caused by the disaster was rarely incorporated into the analysis.

In fact, Tsubokura et $a l^{27}$ showed that in evacuees living in temporary housing $(n=200)$ in Fukushima prefecture, body weight, BMI, waist circumference and HbAlc were significantly increased but serum HDLC was decreased at 6 months after the disaster compared to those observed before the disaster. Although our results are partially consistent with results of that study, there were no control participants (non-evacuees) and

Table 3 Multiple linear regression analysis of variables for examining mean change in body weight between phase 1 and phase 2 (stepwise selection methods)

\begin{tabular}{|c|c|c|c|c|c|c|}
\hline \multirow[b]{2}{*}{ Variable } & \multicolumn{2}{|l|}{ Model 1} & \multicolumn{2}{|l|}{ Model 2} & \multicolumn{2}{|l|}{ Model 3} \\
\hline & $\beta$ (SE) & p Value & $\beta$ (SE) & p Value & $\beta$ (SE) & p Value \\
\hline REL vs non-REL (2012) & $0.55(0.06)$ & $<0.001$ & $0.55(0.06)$ & $<0.001$ & $0.56(0.06)$ & $<0.001$ \\
\hline Sex & $-0.31(0.06)$ & $<0.001$ & $-0.22(0.07)$ & 0.001 & $-0.30(0.06)$ & $<0.001$ \\
\hline Age (2011) & $-0.01(0.00)$ & 0.031 & Not included & & Not included & \\
\hline Body weight (2011) & & & $-0.01(0.00)$ & 0.038 & Not included & \\
\hline SBP $(2011)$ & & & $-0.01(0.00)$ & $<0.001$ & $-0.01(0.00)$ & $<0.001$ \\
\hline Current smokers vs not current smokers (2011) & & & & & Not included & \\
\hline $\begin{array}{l}\text { Low physical activity vs normal physical activity } \\
\text { (2011) }\end{array}$ & & & & & Not included & \\
\hline $\begin{array}{l}\text { Psychological distress vs no psychological } \\
\text { distress (2011) }\end{array}$ & & & & & Not included & \\
\hline Unemployment vs not unemployment (2011) & & & & & $-0.17(0.07)$ & 0.023 \\
\hline Economic deprivation vs usual (2011) & & & & & Not included & \\
\hline
\end{tabular}


no psychological or socioeconomic survey in that study. Therefore, the interpretation of changes in the cardiometabolic risk factors in Tsubokura's report may have limitations. In Italy, factory workers who experienced a catastrophic earthquake showed significant increases in body weight and serum levels of TC and TG 2 months after the disaster compared to those of unexposed participantts. ${ }^{26}$ This increase in body weight after the disaster is comparable with our results, but serum levels of TC and TG were not significantly changed in our study period. The major differences between our study and the previous study were the timing of the study and the degree/type of disaster. In the Italian study, data obtained before and 2 months after the earthquake were compared, whereas changes in metabolic profile from 8 to 18 months after the earthquake and subsequent tsunami were investigated in our study. In terms of our study, it may be plausible that even more than 1 year after the complex disaster, body weight/waist circumference and lipid levels would have deteriorated consistently in adults in the general population who experienced tsunami-related evacuation.

Subjects in our study who suffered earthquake damage only without serious tsunami damage (non-REL group) showed no changes in metabolic profiles during the study period. This group also seemed to experience significantly better conditions for lifestyle, mental health and socioeconomic status compared to those for the REL participants. Thus, this study suggests that the mental and socioeconomic problems caused by the tsunami may lead to a continuous deterioration in cardiometabolic risks and impaired health status. The extent of psychological and social disruption caused by the major tsunami in the affected population may thus be greater than that caused by the earthquake itself.

\section{Mental health and socioeconomic status}

While previous studies have shown worsening mental health after catastrophic disasters, ${ }^{28}{ }^{29}$ few studies have focused on postdisaster psychological distress as represented by disaster-related REL. Yokoyama $e t a l^{14}$ showed a relationship between the extent of REL and the degree of deterioration in mental health problems. In our study, there was a significant difference in mean body weight and waist circumference changes during the two study phases between the REL group and the non-REL group. The percentage of participants with psychological distress as evaluated by the K6 score was significantly higher in the REL group than in the non-REL group. In addition, as shown in table 1, participants with REL were more likely to have lost jobs and were more likely to have experienced a serious economic crisis. It may therefore be possible that disaster-related mental distress and socioeconomic problems worsen metabolic risk factors indirectly by motivating harmful behaviours such as cigarette smoking and physical inactivity. In addition, several recent studies have suggested that individuals with higher psychological stress were more likely to exhibit less healthy dietary behaviours with higher body weight ${ }^{30}$ and higher cardiometabolic risk. ${ }^{32}$

\section{Weight gain and HDLC}

This study showed significant weight gain and increased waist circumference together with decreased levels of serum HDLC during the convalescent phase after the disaster in the REL group. There are several possible causes for the decreased serum HDLC level including physical inactivity, smoking, weight gain, elevated TG, end-stage renal disease and type 2 diabetes mellitus. ${ }^{33}$ Our results showed significant differences in body weight and waist circumference changes and in physical activity patterns and smoking status between the REL group and the non-REL group. Thus, it is likely that these factors contributed to the decrease in serum HDLC levels in the REL group. The possible mechanisms for decreased HDLC in the REL group are considered to be as follows. Increased visceral fat increases free fatty acid levels, elevates serum levels of TG and very lowdensity lipoprotein, and impairs bioactivity of cholesterol ester transfer protein and hepatic lipase. Our study also showed lower physical activity levels in participants in the REL group. Physical training has been reported to improve lipoprotein lipase activity and insulin resistance with decreases in cholesterol ester transfer protein activity and serum TG. Inversely, physical inactivity may decrease the serum level of HDLC. In addition, the percentage of current smokers was higher in the REL group. Chelland Campbell et $a \hat{l}^{4}$ suggested that cigarette smoking stimulates the sympathetic nervous system and thus activates free fatty acid release. This promotes an increase in circulation of very low-density lipoprotein and LDLC and thus causes lower serum HDLC levels.

\section{Limitations}

This study had several limitations. First, since the initial (phase 1) survey began several months after the disaster, atherosclerotic cardiovascular risk factors including lifestyle and socioeconomic state before the prior disaster could not be determined. Therefore, it is possible that these results were already in a trajectory of worsening body weight gain, increased waist circumference and decreased HDLC levels. However, as shown in table 1, there were close relationships between REL and several disaster-related unhealthy conditions including smoking, physical inactivity, psychological distress and socioeconomic deprivations. In view of these, we believe that REL in this study might be a general marker of a cluster of disaster-related health strains, and that it might also be associated with observed results. Second, although we assessed mental health status using the standard K6 questionnaires only, it is not clear whether $\mathrm{K} 6$ scoring is the best method for determining mental status after this type of major disaster. Of course, there is no doubt that our participants were living in extremely stressful circumstances after the disaster, but we may have needed additional, more detailed mental tests. Third, we were 
unable to adjust for use of medications, especially psychotropic and antihyperlipidaemic drugs, which have implications for cardiometabolic risk factors. In addition, caloric intake, level of income, job status and educational level of the participants before the disaster were not determined. We could not completely exclude the possibility that these unmeasured variables might cause a certain bias in the present results. Fourth, we could not determine whether there was any increase in the incidence of diabetes mellitus, hypertension or atherosclerotic cardiovascular events during this study period. We need to investigate the relationships between these disorders and changes in atherosclerotic cardiovascular risk profiles. Fifth, our participants may not be representative of the general population because three-fourths of the residents did not attend the initial survey in 2011. Those who did not participate in our survey might have serious diseases and be under medical care or they might have been busy mostly due to postdisaster settings. On the other hand, those who took part in our survey tended to be conscious of their health. Finally, onefourth of the participants (approximately 26\%) in the initial survey did not participate in the phase 2 survey. We therefore compared several anthropometric measurements and clinical parameters in the phase 1 survey between non-participants $(\mathrm{n}=2689)$ and participants ( $\mathrm{n}=6528)$ (see online supplementary Table S1). In sex/ age-adjusted analysis, systemic blood pressure was significantly higher in the non-participants than in the participants, and the adjusted proportion of current smokers was higher in the non-participants than in the participants. These results suggest that the non-participants may be unhealthier after the phase 1 study than the participants of the phase 2 study. This bias may thus have led to an underestimation in our results.

\section{CONCLUSION}

During the convalescent phase more than 1 year after the disaster, we found that REL was related to weight gain and decreasing HDLC after the disaster. In the light of these findings, we believe that long-term vigilance for changes in atherosclerotic cardiovascular risk factors and events is warranted after any future devastating disasters.

\section{Author affiliations \\ ${ }^{1}$ Department of International Health, Nagasaki University Graduate School of Biomedical Sciences, Sakamoto, Nagasaki, Japan \\ ${ }^{2}$ Department of Hygiene and Preventive Medicine, Iwate Medical University, School of Medicine, Shiwa-gun, Iwate, Japan \\ ${ }^{3}$ Division of Cardioangiology, Department of Internal Medicine, Iwate Medical University, School of Medicine, Morioka, Iwate, Japan \\ ${ }^{4}$ Iwate Medical University, Morioka, Iwate, Japan \\ ${ }^{5}$ Department of Plastic and Reconstructive Surgery, Iwate Medical University, School of Medicine, Morioka, Iwate, Japan}

Acknowledgements The authors would like to thank the participants in the study as well as their fellow healthcare workers. They also thank Drs. Mikihito Ishiki, Masahiro Ueno, Makoto Koshiyama, Kazuyoshi Itai, Masaki Osawa,
Toru Kuribayashi, Ryohei Sasaki, Nobuyuki Takanashi and Yukari Yokoyama for their useful advice.

Contributors ST was involved in the analysis of data, interpretation of data and drafting of the manuscript. MN was involved in the study concept and design, interpretation of data and the critical revision of the manuscript. YY and KT helped the statistical analyses and participated in the data collection, database design and manuscript review. KS and AO participated in the study design, database design and manuscript review. SK is the chairperson of the RIAS Study and was involved in the study concept and design, critical revision of the manuscript and acquisition of funding.

Funding The study was supported by a Health Labour Sciences Research Grant from the Ministry of Health, Labour, and Welfare of Japan (H23-TokubetsuShitei-002; H24-kenki-sitei-001).

Competing interests None declared.

Patient consent Obtained.

Ethics approval The Ethics Committee of Iwate Medical University (approval no. H23-69).

Provenance and peer review Not commissioned; externally peer reviewed.

Data sharing statement No additional data are available.

Open Access This is an Open Access article distributed in accordance with the Creative Commons Attribution Non Commercial (CC BY-NC 4.0) license, which permits others to distribute, remix, adapt, build upon this work noncommercially, and license their derivative works on different terms, provided the original work is properly cited and the use is non-commercial. See: http:// creativecommons.org/licenses/by-nc/4.0/

\section{REFERENCES}

1. The National Police Agency, Japan. Damage Situation and Police Countermeasures associated with 2011Tohoku district-off the Pacific Ocean Earthquake. 2015. [updated 10 December 2015; cited 3 January 2016]. https://www.npa.go.jp/archive/keibi/biki/higaijokyo_ e.pdf

2. Matsubara $\mathrm{C}$, Murakami $\mathrm{H}$, Imai $\mathrm{K}$, et al. Prevalence and risk factors for depressive reaction among resident survivors after the tsunami following the Great East Japan Earthquake, March 11, 2011. PLoS ONE 2014;9:e109240.

3. Kloner RA, Leor J, Poole WK, et al. Population-based analysis of the effect of the Northridge Earthquake on cardiac death in Los Angeles County, California. J Am Coll Cardiol 1997;30:1174-80.

4. Leor J, Poole WK, Kloner RA. Sudden cardiac death triggered by an earthquake. N Engl J Med 1996;334:413-19.

5. Watanabe H, Kodama M, Okura $\mathrm{Y}$, et al. Impact of earthquakes on Takotsubo cardiomyopathy. JAMA 2005;294:305-7.

6. Tanaka F, Makita S, Ito $\mathrm{T}$, et al. Relationship between the seismic scale of the 2011 northeast Japan earthquake and the incidence of acute myocardial infarction: a population-based study. Am Heart $J$ 2015;169:861-9.

7. Niiyama M, Tanaka F, Nakajima S, et al. Population-based incidence of sudden cardiac and unexpected death before and after the 2011 earthquake and tsunami in Iwate, northeast Japan. J Am Heart Assoc 2014;3:e000798.

8. Nakamura M, Tanaka F, Nakajima S, et al. Comparison of the incidence of acute decompensated heart failure before and after the major tsunami in Northeast Japan. Am J Cardiol 2012;110:1856-60.

9. Omama S, Yoshida Y, Ogasawara K, et al. Influence of the great East Japan earthquake and tsunami 2011 on occurrence of cerebrovascular diseases in Iwate, Japan. Stroke 2013;44:1518-24.

10. Nakagawa I, Nakamura K, Oyama M, et al. Long-term effects of the Niigata-Chuetsu earthquake in Japan on acute myocardial infarction mortality: an analysis of death certificate data. Heart 2009;95:2009-13.

11. Peters MN, Katz MJ, Moscona JC, et al. Effect of Hurricane Katrina on chronobiology at onset of acute myocardial infarction during the subsequent three years. Am J Cardiol 2013;111:800-3.

12. Dimsdale JE. Psychological stress and cardiovascular disease. J Am Coll Cardiol 2008;51:1237-46.

13. Japan Science and Technology Agency. The Great East Japan Earthquake information from official websites [Internet]. 2011. (cited 12 Octpber 2014). http://www.jst.go.jp/saigai.html

14. Yokoyama Y, Otsuka K, Kawakami N, et al. Mental health and related factors after the Great East Japan earthquake and tsunami. PLOS ONE 2014;9:e102497. 
15. Seino $\mathrm{Y}$, Nanjo K, Tajima N, et al. Report of the committee on the classification and diagnostic criteria of diabetes mellitus. J Diabetes Investig 2010;1:212-28.

16. Ministry of health, labor and welfare, Japan. Exercise guidelines for good health 2006. 2006. http://www.mhlw.go.jp/bunya/kenkou/ undou02/pdf/data.pdf (accessed 3 Aug 2015).

17. Murakami H, Yoshimura E, Ishikawa-Takata $\mathrm{K}$, et al. Validity and reproducibility of a physical activity questionnaire used for health surveying among victims of the Great East Japan Earthquake. Nihon Koshu Eisei Zasshi 2013;60:222-30.

18. Kessler RC, Barker PR, Colpe LJ, et al. Screening for serious mental illness in the general population. Arch Gen Psychiatry 2003;60:184-9.

19. Sakurai K, Nishi A, Kondo K, et al. Screening performance of K6/ $\mathrm{K} 10$ and other screening instruments for mood and anxiety disorders in Japan. Psychiatry Clin Neurosci. 2011;65:434-41.

20. Soldatos CR, Dikeos DG, Paparrigopoulos TJ. Athens Insomnia Scale: Validation of an instrument based on ICD-10 criteria. $J$ Psychosom Res 2000;48:555-60.

21. Okajima I, Nakajima S, Kobayashi M, et al. Development and validation of the Japanese version of the Athens Insomnia Scale. Psychiatry Clin Neurosci 2013;67:420-5.

22. Kario $\mathrm{K}$, Matsuo $\mathrm{T}$, Kobayashi $\mathrm{H}$, et al. Earthquake-induced potentiation of acute risk factors in hypertensive elderly patients: possible triggering of cardiovascular events after a major earthquake. J Am Coll Cardiol 1997;29:926-33. [published Online First: 1 April 1997]

23. Minami J, Kawano Y, Ishimitsu T, et al. Effect of the Hanshin-Awaj earthquake on home blood pressure in patients with essential hypertension. Am J Hypertens 1997;10:222-5.

24. Satoh M, Kikuya M, Ohkubo T, et al. Acute and subacute effects of the great East Japan earthquake on home blood pressure values. Hypertension 2011;58:e193-4.
25. Ogawa S, Ishiki M, Nako K, et al. Effects of the Great East Japan Earthquake and huge tsunami on glycaemic control and blood pressure in patients with diabetes mellitus. BMJ Open 2012;2: e000830.

26. Trevisan M, Celentano E, Meucci C, et al. Short-term effect of natural disasters on coronary heart disease risk factors. Arteriosclerosis 1986:6:491-4.

27. Tsubokura M, Takita M, Matsumura $\mathrm{T}$, et al. Changes in metabolic profiles after the Great East Japan Earthquake: a retrospective observational study. BMC Public Health 2013;13:267.

28. Galea S, Nandi A, Vlahov D. The epidemiology of post-traumatic stress disorder after disasters. Epidemiol Rev 2005;27:78-91.

29. Van Griensven F, Chakkraband ML, Thienkrua W, et al. Mental health problems among adults in tsunami-affected areas in southern Thailand. JAMA 2006;296:537-48.

30. Moore CJ, Cunningham SA. Social position, psychological stress, and obesity: a systematic review. J Acad Nutr Diet 2012;112:518-26.

31. Harding JL, Backholer K, Williams ED, et al. Psychosocial stress is positively associated with body mass index gain over 5 years: evidence from the longitudinal AusDiab study. Obesity (Silver Spring) 2014;22:277-86.

32. Winning A, Glymour MM, McCormick MC, et al. Psychological distress across the life course and cardiometabolic risk: findings from the 1958 British Birth Cohort Study. J Am Coll Cardiol 2015;66:1577-86.

33. Hayden MR, Tyagi SC. Isolated low high density lipoproteincholesterol (HDL-C): implications of global risk reduction. Case report and systematic scientific review. Cardiovasc Diabetol 2005;4:1

34. Chelland Campbell S, Moffatt RJ, Stamford BA. Smoking and smoking cessation-the relationship between cardiovascular disease and lipoprotein metabolism: a review. Atherosclerosis 2008;201:225-35 